\title{
Polydatin Attenuates Cisplatin-Induced Acute Kidney Injury by Inhibiting Ferroptosis
}

\author{
Lu Zhou $\mathbb{D}^{1}{ }^{1}$ Peng Yu, ${ }^{1,2}$ Ting-ting Wang, ${ }^{1}$ Yi-wei Du, ${ }^{1}$ Yang Chen, ${ }^{1}$ Zhen Li, ${ }^{1,2}$ \\ Man-lin He, ${ }^{1,2}$ Lan Feng, ${ }^{1}$ Hui-rong Li, ${ }^{1}$ Xiao Han, ${ }^{1,2}$ Heng Ma $\mathbb{D}^{3},{ }^{3}$ and Hong-bao Liu $\mathbb{D}^{1}$ \\ ${ }^{1}$ Department of Nephrology, Tangdu Hospital, Fourth Military Medical University, Xi'an 710038, China \\ ${ }^{2}$ Shaanxi University of Chinese Medicine, Xianyang 712046, China \\ ${ }^{3}$ Department of Physiology and Pathophysiology, School of Basic Medical Sciences, Fourth Military Medical University, \\ Xi'an 710032, China
}

Correspondence should be addressed to Heng Ma; hengma@fmmu.edu.cn and Hong-bao Liu; xjsnlhb@fmmu.edu.cn

Received 29 August 2021; Revised 10 December 2021; Accepted 11 December 2021; Published 15 January 2022

Academic Editor: Mario Zoratti

Copyright (c) $2022 \mathrm{Lu}$ Zhou et al. This is an open access article distributed under the Creative Commons Attribution License, which permits unrestricted use, distribution, and reproduction in any medium, provided the original work is properly cited.

Cisplatin is widely used in the treatment of solid tumors, but its application is greatly limited due to its nephrotoxicity; thus, there is still no effective medicine for the treatment of cisplatin-induced acute kidney injury (Cis-AKI). We previously identified that polydatin (PD) exerts nephroprotective effects by antioxidative stress in AKI models. Recent evidence suggests that oxidative stress-induced molecular events overlap with the process of ferroptosis and that there are common molecular targets, such as glutathione (GSH) depletion and lipid peroxidation. Nevertheless, whether the nephroprotective effect of PD is related to antiferroptosis remains unclear. In this study, the inhibitory effect of PD on ferroptosis was observed in both cisplatin-treated HK2 cells $(20 \mu \mathrm{M})$ in vitro and a Cis-AKI mouse model $(20 \mathrm{mg} / \mathrm{kg}$, intraperitoneally) in vivo, characterized by the reversion of excessive intracellular free iron accumulation and reactive oxygen species (ROS) generation, a decrease in malondialdehyde (MDA) content and GSH depletion, and an increase in glutathione peroxidase-4 (GPx4) activity. Remarkably, PD dosedependently alleviated cell death induced by the system $\mathrm{Xc}^{-}$inhibitor erastin $(10 \mu \mathrm{M})$, and the effect of the $40 \mu \mathrm{M}$ dose of PD was more obvious than that of ferrostatin-1 $(1 \mu \mathrm{M})$ and deferoxamine (DFO, $100 \mu \mathrm{M})$, classical ferroptosis inhibitors. Our results provide insight into nephroprotection with $\mathrm{PD}$ in Cis-AKI by inhibiting ferroptosis via maintenance of the system Xc ${ }^{-}-$ GSH-GPx4 axis and iron metabolism.

\section{Introduction}

Cisplatin is a chemotherapeutic agent widely used to treat various malignancies, and its application is greatly limited due to its nephrotoxicity, including its onset of acute kidney injury (AKI) [1]. Undoubtedly, effective drugs to prevent cisplatin-induced AKI (Cis-AKI) in clinical practice are in urgent demand. Therefore, it is very important to further investigate the pathophysiology of Cis-AKI and its effective therapeutic drugs.

Several studies have shown that DNA damage, oxidative stress, inflammation, vascular dysfunction, and mitochondrial damage may be involved in the pathogenesis of CisAKI [1]. Recently, several studies [2-6] have suggested that cisplatin treatment leads to excessive lipid peroxidation, ferritinophagy-mediated free iron release, and a decrease in the activity of glutathione peroxidase-4 (GPx4), indicating the close link between ferroptosis and Cis-AKI. Hence, ferroptosis intervention may be an effective strategy to attenuate Cis-AKI; nevertheless, there have not been specific drugs against ferroptosis in clinical application to date.

Polydatin $\left(\mathrm{PD}, \mathrm{C}_{20} \mathrm{H}_{22} \mathrm{O}_{8}\right.$ ) is a natural active ingredient extracted from the dried roots of the perennial herb Polygonum cuspidatum Sieb. et Zucc., which might play a potential therapeutic role in various kidney disorders, such as AKI [7-16], diabetic nephropathy [17-24], lupus nephritis [25], and hyperuricemia [26-28], and has been used to treat patients with irritable bowel syndrome in Europe [29]; moreover, PD has been shown to exhibit mechanisms to antioxidant stress, anti-inflammation, and antifibrosis 
characteristics; improvement of mitochondrial damage; and regulation of autophagy $[9,30,31]$. Resveratrol, an aglycone of PD, has been verified to ameliorate myocardial and liver damage caused by iron overload [32-34]; moreover, it has been confirmed that resveratrol can reduce iron load in hemodialysis patients in clinical studies [35]. Recent studies have noted that PD [36] or resveratrol $[37,38]$ can inhibit ferroptosis to ameliorate myocardial ischemia-reperfusion injury and brain injury. However, the role of PD in CisAKI is still unclear. Our previous studies have demonstrated that PD, which is capable of increasing the levels of glutathione (GSH) and GPx and reducing the content of malondialdehyde (MDA), plays a renal protective role in the renal ischemia-reperfusion injury model through antioxidative stress and anti-inflammatory mechanisms [13-15]. A series of molecular events induced by oxidative stress overlap with ferroptosis processes after AKI, with common molecular targets such as lipid peroxidation and GSH depletion. All of the evidence above strongly indicates that PD may be a latent therapeutic compound against ferroptosis in AKI.

Considering that the antiferroptotic effect of PD in AKI has not yet been clearly reported, the purpose of this study was to investigate the relationship between the nephroprotective effect and antiferroptotic role in Cis-AKI to provide solid evidence for the research and development of nephroprotective agents for clinical AKI treatment.

\section{Materials and Methods}

2.1. Chemicals and Reagents. Cisplatin, dimethyl sulfoxide (DMSO), Hoechst 33342 fluorescent staining kit (B2261), and dihydroethidium (DHE) fluorescent staining kit (D7008) were purchased from Sigma-Aldrich (St. Louis, MO, USA). Polydatin (PD, MB5448) and ferrostatin-1 (Fer1, MB4718) were acquired from Meilun Biotech (Dalian, China). Deferoxamine (DFO, HY-B0988) was purchased from MedChemexpress LLC (Princeton, USA). Lipid peroxidation malondialdehyde (MDA) assay kit (S0131M), reactive oxygen species (ROS) assay kit (S0033M), DCFH-DA fluorescent probe assay (S0033S), mitochondrial membrane potential assay kit with JC-1 (C2006), and cellular glutathione peroxidase (GPX) assay kit with NADPH (S0056) were purchased from Beyotime Biotechnology (Shanghai, China). CheKine ${ }^{\mathrm{TM}}$ Reduced Glutathione (GSH) Colorimetric Assay Kit (KTB1600) was purchased from Abbkine (Wuhan, China). 4 Hydroxynonenal (4HNE) ELISA assay kit was purchased from Yanqi Biotech (Shanghai, China). Anti-4HNE antibody (ab46545) and lipid hydroperoxide (LPO) assay kit (ab133085) was acquired from Abcam (ON, Canada). Phosphate-buffered saline (PBS), Dulbecco's modified Eagle's medium/nutrient mixture F-12 (DMEM/F-12), fetal bovine serum (FBS), Hanks' balanced salt solution (HBSS), cell counting kit (CCK-8), DAPI fluorescence staining kit (G1012), and fluorescein (FITC) Tunel cell apoptosis detection kit (TUNEL, G1501) were purchased from Servicebio Technology (Wuhan, China). FeRhoNox-1 fluorescent probe (MX4558) was purchased from Maokang Biotech (Shanghai, China). HK2(GDC0152) was purchased from China Center for Type Culture Collection (Wuhan, China).
2.2. Animals and Experimental Protocol. All animal experiments were conducted in strict accordance with the Guidelines of Health and guidelines for use and were permitted by the Scientific Investigation Committee of the Fourth Military Medical University. Male C57BL/6 mice (8-10 weeks of age, weight 20-25 g) were purchased from Experimental Animal Center of the Fourth Military Medical University (Xi'an, China) and bred in an experimental animal room of SPF grade. They were randomly divided into four groups: control (equivalent saline containing 1\% DMSO) group $(n=5)$, cisplatin $(20 \mathrm{mg} / \mathrm{kg}$ dissolved in saline) only group $(n=7)$, cisplatin + polydatin $(40 \mathrm{mg} / \mathrm{kg}$ dissolved in $1 \%$ DMSO) group $(n=7)$, and cisplatin + Fer-1 $(5 \mathrm{mg} / \mathrm{kg}$ dissolved in $1 \%$ DMSO) group $(n=7)$ were administered intraperitoneally. Mice were injected with cisplatin once; PD or Fer- 1 was given $1 \mathrm{~h}$ before and $24 \mathrm{~h}$ after cisplatin. Animals were ethically sacrificed by dislocating their spines at $48 \mathrm{~h}$ after cisplatin injection, and whole blood and kidneys were collected for further analysis.

2.3. Blood Physiochemical Assays. The whole blood drawn from the retroocular vein plexus was centrifuged at $4^{\circ} \mathrm{C}$, $4000 \mathrm{rpm}$, for $10 \mathrm{~min}$ to acquire the serum sample. The level of blood urea nitrogen (BUN) and serum creatinine (Scr) was measured according to manufacturer's instructions using the urea determination kit (C013-2-1, Nanjing Jiancheng, China) and creatinine determination kit (C011-2-1, Nanjing Jiancheng, China), respectively.

\subsection{Renal Tissue Histopathological, Immunohistochemistry} (IHC), and Terminal Deoxynucleotidyl Transferase dUTP Nick-End Labeling (TUNEL) Assay. Fresh renal tissues were washed with ice-cold stroke-physiological saline solution, fixed overnight with $10 \%$ neutral buffered formalin, and then paraffin-embedded, followed cut into a thickness of $4 \mu \mathrm{m}$ sections, which were used for hematoxylin-eosin (H\&E) staining, or TUNEL fluorescent staining, or 4HNE immunohistochemistry according to manufacturer's instructions. Evaluation of histological score of kidney injuries (HSK) was performed by a renal pathologist under the blinded manner. The kidney sections representing a minimum of 100 fields of at least 5 mice from each group were semiquantitatively assessed. HSK was scored using a 4point quantitative scale, as previously described by us [13, 14]: 0 represented normal histology; 1 represented mild injuries [nuclear lost (necrosis) less than $1 / 3$ tubular section]; 2 represented moderate damage [more than $1 / 3$ and less than $2 / 3$ of a tubular cross section shows nuclear loss (necrosis)]; 3 represented the severe damage [more than $2 / 3$ of nuclear loss (necrosis) per tubular cross-section]. We calculated the total score for each kidney slice and added all 10 scores to a maximum possible injury score of 30 .

2.5. Mitochondrial Morphology Observation by Transmission Electron Microscopy (TEM). Simply, $1 \mathrm{~mm}^{3}$ of the fresh renal cortex was removed and quickly placed in a TEM fixative at $4^{\circ} \mathrm{C}$. Tissues were embedded and cut into ultrathin sections at $60-80 \mathrm{~nm}$, and then, uranium lead double staining was 
performed. The ultrastructure of the kidney was observed by TEM, and the images were collected.

2.6. Determination of Lipid Peroxidesand Lipid Peroxidation. The lipid peroxide (LPO) and MDA levels in supernatants from both the renal tissues and cultured HK-2 cells were measured using a commercially available kit, and absorbance was measured at $500 \mathrm{~nm}$ and $535 \mathrm{~nm}$ using a spectrophotometer, respectively, according to manufacturer's instructions [16]. In addition, HK-2 cells were also incubated with C11-BODIPY581/591 (D3861, Invitrogen, USA) and DAPI in flow cytometry buffer (2\% FCS, 2 mM EDTA in PBS) at $37^{\circ} \mathrm{C}$ for 10 to $30 \mathrm{~min}$ in darkness. The cells were subsequently washed with PBS and resuspended in FACS buffer for flow cytometry (excitation $480 \mathrm{~nm}$, emission $510 \mathrm{~nm}$ and $590 \mathrm{~nm}$ ) [39]. BODIPY-positive cells among DAPInegative cells were analyzed as compared to a control sample using BODIPY measurement.

2.7. Determination of GSH Levels and GPx Activity. The GSH levels and GPx4 activity in supernatants from both the renal tissues and cultured HK-2 cells were determined using CheKine ${ }^{\mathrm{TM}}$ Reduced GSH Colorimetric Assay Kit and Cellular Glutathione Peroxidase Assay Kit with NADPH, respectively, according to manufacturer's instructions [5]. The absorbance of each sample was measured at $412 \mathrm{~nm}$ for GSH and at $340 \mathrm{~nm}$ for GPx4 using a spectrophotometer. The GSH concentration and GPx4 activity were calculated according to the standard curve. A total of three independent repeats were performed.

2.8. Cell Culture and Treatment. Human proximal tubular epithelial cells (HK-2 cells) were obtained from the China Center for Type Culture Collection (GDC0152, Wuhan, China), grown in DMEM/F-12 supplemented with $10 \%$ heat-inactivated FBS, $100 \mathrm{U} / \mathrm{ml}$ penicillin, and $100 \mu \mathrm{g} / \mathrm{ml}$ streptomycin in a humidified atmosphere of $5 \% \mathrm{CO}_{2}$ and $95 \%$ air at $37^{\circ} \mathrm{C}$. Exponentially growing $\mathrm{HK}-2$ cells were seeded at 2 to $4 \times 10^{5}$ cells/well in six-well culture plates and cultured for 1 day before each experiment. Then, the cells were divided into four groups: control group, cisplatin $(20 \mu \mathrm{M})$ group, cisplatin $+\mathrm{PD}(40 \mu \mathrm{M})$ group, and cisplatin + Fer-1 $(1 \mu \mathrm{M})$ group.

2.9. Cell Viability Assay. HK-2 cells were cultured without or with different concentrations of PD $(10,20$, and $40 \mu \mathrm{M})$, DFO $(100 \mu \mathrm{M})$ or Fer-1 $(1 \mu \mathrm{M})$ in presence or absence of cisplatin $(20 \mu \mathrm{M})$, and the cell viability was measured by CCK-8 according to manufacturer's instructions. So, as to evaluate the effect of PD on ferroptosis, in some experiments, we replaced cisplatin with erastin $(10 \mu \mathrm{M})$.

2.10. Determination of Intracellular Labile Iron. The cellular labile iron pool (LIP) is a compartment of nonferritin-bound iron that can generate oxygen radicals via the Fenton reaction resulting in oxidative stress and cell injury, including ferroptosis. Intracellular LIP in the kidney tissue was measured by electron paramagnetic resonance (EPR) spectroscopy (Magnettech GmbH, Germany) as described in Woodmansee and Imlay [40] with modifications. Mice kidney samples were homogenized in $10 \mathrm{mM}$ Tris- $\mathrm{HCl}$ buffer, $120 \mathrm{mM} \mathrm{KCl} \mathrm{(pH} \mathrm{7.4),} \mathrm{and} 1 \mathrm{mM}$ DFO. Samples were incubated on ice for $1 \mathrm{~h}$ and then rapidly frozen with liquid nitrogen in $4 \mathrm{~mm}$ O.D. EPR tubes. Under the condition of central magnetic field $1600 \mathrm{G}$, sweep width $800 \mathrm{G}$, microwave frequency $9.75 \mathrm{GHz}$, microwave power $20 \mathrm{~mW}$, modulation frequency $50 \mathrm{kHz}$, modulation amplitude $4.759 \mathrm{G}$, and time constant $81.92 \mathrm{~ms}$, EPR spectra were recorded. All samples were analyzed three times; the sample was removed from the EPR cavity each time and then repositioned within the cavity prior to initiating spectral scanning.

The content of labile iron in HK-2 cells was monitored using selective $\mathrm{Fe}^{2+}$ fluorescent probe FeRhoNox-1, calcein-chelatable cytosolic LIP assay, and Iron Assay Kit (Sigma-Aldrich, MAK025). FeRhoNox-1, which is a turnon fluorescent probe specific for the detection of labile iron $\mathrm{Fe}^{2+}$, was used to detect intracellular LIP, and the cellular distribution of FeRhoNox-1 was consistent with Golgi [41]. HK-2 cells were grown to confluence in $35 \mathrm{~mm}$ laser confocal petri dishes in DMEM, and PD $(40 \mu \mathrm{M})$ or Fer-1 $(1 \mu \mathrm{M})$ was added in the absence or presence of cisplatin $(20 \mu \mathrm{M})$. Cells were incubated with $5 \mu \mathrm{M}$ FeRhoNox-1 for $1 \mathrm{~h}$ prior to assays. Cells were washed twice with PBS before staining nuclei with Hoechst 33342. The fluorescence was immediately observed with a confocal laser-scanning microscope (CLSM, ECLIPSE Ti, Nikon, Tokyo, Japan).

Calcein acetoxymethyl ester (Calcein-AM, Corning Inc., Corning, NY, USA), which is the most widely adopted labile iron fluorescent probe, was used to detect cytosolic LIP. Calcein-AM is a nonfluorescent lipophilic ester that passes through the cellular membrane and reacts with cytosolic unspecific esterases to produce calcein, a fluorochromic alcohol that chelates labile iron under quenching of the green fluorescence [42]. Briefly, the cells were loaded at $37^{\circ} \mathrm{C}$ with $2 \mu \mathrm{M}$ calcein for $30 \mathrm{~min}$ and then washed with HBSS. DFO was added at a final concentration of $100 \mu \mathrm{M}$ to remove iron from calcein, leading to dequenching. Fluorescence changes with the addition of DFO were used for indirect measurement of the LIP. Fluorescence was measured at $485 \mathrm{~nm}$ excitation and $535 \mathrm{~nm}$ emissions with a fluorescence plate reader.

In addition, intracellular level of ferrous $\left(\mathrm{Fe}^{2+}\right)$ iron was also determined using an iron assay kit from Sigma-Aldrich (MAK025), according to manufacturer's instructions. Absorbance was measured at $593 \mathrm{~nm}$ using a microplate reader.

2.11. Mitochondrial Membrane Potential (MMP) by Florescent JC-1. The MMP was measured using JC-1 fluorescent probes, a monomer present in the cytosol (staining green), which also aggregated in the normally polarized mitochondria (which stains red). However, in damaged and depolarized mitochondria, JC-1 exists as a monomer and stains the cytosol green. The change of fluorescence emission from red to green indicates mitochondrial depolarization. HK-2 cells were incubated with $5 \mu \mathrm{mol} / \mathrm{l} \mathrm{JC}-1$ for $15 \mathrm{~min}$ at $37^{\circ} \mathrm{C}$. A minimum of 104 cells per sample was analyzed using a FACS Calibur flow cytometer (Becton Dickinson, BD Biosciences, USA). Data were analyzed using 
BD FACSuite software. The percentage of cells with abnormally low MMP (i.e., green fluorescence) was also measured.

2.12. ROS Detection. Dihydroethidium (DHE) fluorescence was used to detect the ROS levels in the renal tissues. The renal tissues were immersed in saccharose $(30 \% w / v)$, embedded at the optimal cutting temperature (OCT), and stored at $-20^{\circ} \mathrm{C}$ until immunofluorescence assay. The OCT blocks were cut into $10 \mu \mathrm{m}$ in a cryostat and mounted on polylysine-coated glass slides. Tissue sections were incubated in $10 \mu \mathrm{M} \mathrm{DHE}$ for $30 \mathrm{~min}$ at $37^{\circ} \mathrm{C}$ in a humidified chamber protected from light, then incubated with DAPI solution at room temperature for $10 \mathrm{~min}$, kept in a dark place. In the presence of superoxide anion, DHE is oxidized to ethidium, which produces a bright red fluorescence. After washing with PBS, sections were mounted and visualized by CLSM.

The intracellular ROS levels of cells were monitored using the DCFH-DA fluorescent probe assay. After entering the cells, DCFH-DA is hydrolyzed by esterases to $2^{\prime}, 7^{\prime}$ -dichlorofluorescin (DCFH), which is captured in the cell. In the presence of ROS, the nonfluorescent DCFH is oxidized and turns into highly fluorescent $2^{\prime}, 7^{\prime}$-dichlorofluorescein (DCF). HK-2 cells were seeded on $35 \mathrm{~mm}$ laser confocal petri dishes at a density of $1.0 \times 10^{6}$ cells per well and cultured at $37^{\circ} \mathrm{C}$ for $24 \mathrm{~h}$ with $5 \% \mathrm{CO}_{2}$. The cells were treated with PD $(40 \mu \mathrm{M})$ or Fer-1 $(1 \mu \mathrm{M})$ for $24 \mathrm{~h}$ in the absence or presence of cisplatin $(20 \mu \mathrm{M})$. The cell culture medium was replaced with DCFH-DA. After incubation for $20 \mathrm{~min}$ in dark at $37^{\circ} \mathrm{C}$, the cells were rinsed using PBS three times to remove the extracellular DCFH-DA. Hoechst 33342 (blue) was added at $37^{\circ} \mathrm{C}$ for $5 \mathrm{~min}$ following manufacturer's instruction. All procedures were done in the dark, and the samples were observed by CLSM.

2.13. Statistical Analysis. Data were presented as the mean \pm standard deviation (SD). Differences between the data means were compared by use of Student's $t$ test and oneway analysis of variance (ANOVA) followed by Dunnett's multiple comparison tests using the SPSS statistical software package (SPSS, Inc., Chicago, IL, USA), and $P$ value of less than 0.05 was considered to be statistically significant.

\section{Results}

3.1. Effects of PD on Attenuating Renal Injury in Cis-AKI Mice. We initially evaluated the role of PD in Cis-AKI and compared it with ferrostatin-1 (Fer-1), a specific ferroptosis inhibitor. To this end, PD $(40 \mathrm{mg} / \mathrm{kg})$ or Fer$1(5 \mathrm{mg} / \mathrm{kg})$ was intraperitoneally injected $1 \mathrm{~h}$ before cisplatin injection and then reinjected $24 \mathrm{~h}$ after cisplatin injection. The Cis-AKI mice were killed $48 \mathrm{~h}$ after cisplatin injection (Figure 1(a)).

Compared with that of the control group, the body weight of Cis-AKI mice was significantly decreased, whereas the body weight of Cis-AKI mice pretreated with PD was obviously improved (Figure 1(b)). Cisplatin treatment resulted in an increase in serum biochemical parameters such as blood urea nitrogen (BUN, Figure 1(c)) and serum creatinine (Scr, Figure 1(d)). Both, PD and Fer-1 treatment, showed suppression in cisplatin-induced injury (Figures 1(c) and $1(\mathrm{~d}))$. Histological examinations were also assessed at $48 \mathrm{~h}$ after cisplatin treatment. As expected, compared with control kidneys from vehicle-treated mice, cisplatin increased the histological score of kidney (HSK) value (Figure 1(e)) and the number of apoptotic cells in the TUNEL assay (Figure 1(f)). Conversely, pretreatment with PD or Fer-1 significantly reduced HSK and TUNELpositive cells (Figures 1(e) and 1(f)).

\subsection{Effects of PD on Inhibiting Ferroptosis in Cis-AKI Mice.} We found that PD have nephroprotective effects similar to Fer-1, however, that much higher doses of PD were used, which prompted us to further explore the impact of $\mathrm{PD}$ on ferroptosis in Cis-AKI mice. For this purpose, ultrastructural shifts and lipid peroxidation levels (a key feature of ferroptosis) were first determined. TEM observation showed no significant changes in the mitochondrial structure of the renal tissue in vehicle-treated mice (Figure 2(a)). In CisAKI mice, ferroptosis-associated mitochondrial changes, such as increased membrane density, reduced mitochondrial volume, and decreased or absent mitochondrial cristae, were observed in renal tissue (Figure 2(a)), which were significantly alleviated to a similar degree by pretreatment with $40 \mathrm{mg} / \mathrm{kg}$ PD or $5 \mathrm{mg} / \mathrm{kg}$ Fer-1 (Figure 2(a)).

Lipid peroxidation is the key driver of ferroptosis. Detection of the lipid peroxidation markers 4-hydroxynonenal (4HNE, Figure 2(b)), MDA (Figure 2(c)), and lipid peroxides (LPOs, Figure $2(\mathrm{~d})$ ) revealed that cisplatin could induce the increase of $4 \mathrm{HNE}, \mathrm{MDA}$, and LPOs levels in the kidneys, and the levels of $4 \mathrm{HNE}, \mathrm{MDA}$, and LPOs were significantly decreased by PD or Fer-1 pretreatment (Figures 2(b)2(d)), indicating that PD is able to reverse the aberrant lipid peroxidation occurring during Cis-AKI.

Depletion of GSH or inhibition of the GSH-dependent antioxidant enzyme GPx4 is also a critical feature of ferroptosis. Inspired by the above data, we also examined the level of GSH and the activity of GPx4. The data showed that the GSH level (Figure 2(e)) and GPx4 activity (Figure 2(f)) decreased $48 \mathrm{~h}$ after Cis-AKI, which could be rescued by PD or Fer-1 pretreatment (Figures 2(e) and 2(f)).

Ferroptosis depends on excess accumulation of free iron, which is an essential component of lipid peroxidation. Therefore, electron paramagnetic resonance (EPR) was used to detect the labile iron pool (LIP) in mouse kidney homogenates in this study. As expected, LIP in kidney homogenates was increased by cisplatin treatment, and PD or Fer-1 pretreatment mitigated the cisplatin-induced increase in LIP (Figure 2(g)).

3.3. Inhibition of PD on Cisplatin-Induced Ferroptosis in HK2 Cells. Since we observed the protective effect of PD against ferroptosis in Cis-AKI mice in vivo, we further verified the effect of PD on erastin $(10 \mu \mathrm{M}$, a specific inducer of ferroptosis)-induced cell toxicity in $\mathrm{HK}-2$ cells in vitro.

The results from the cell counting kit-8 (CCK-8) assay showed that treatment with erastin for $30 \mathrm{~h}$ significantly inhibited cell viability, and treatment with PD dose-dependently alleviated erastin-induced cell death in $\mathrm{HK}-2$ cells compared 


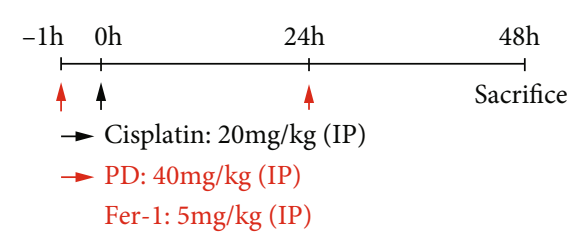

(a)

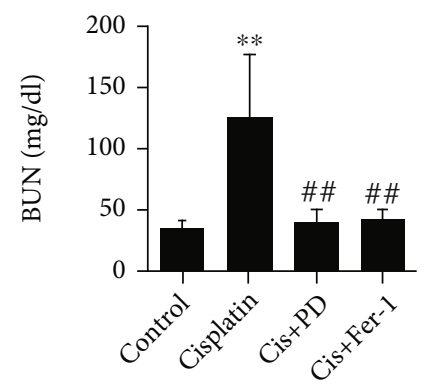

(c)
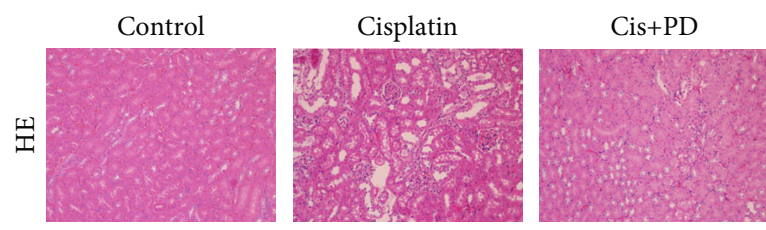

(e)
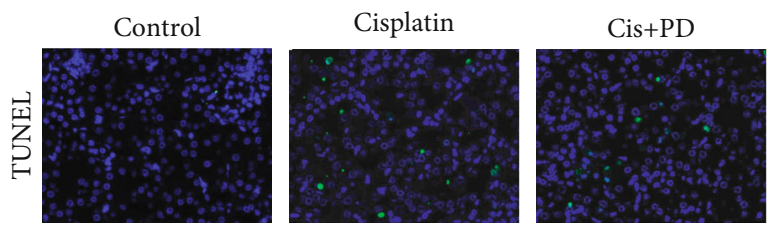

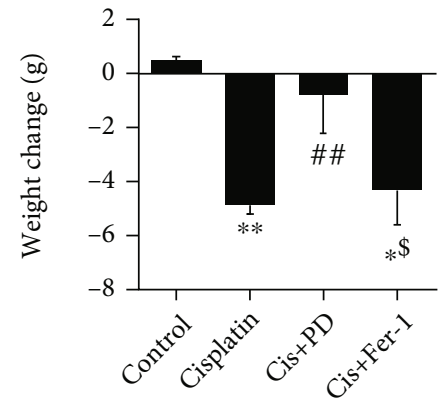

(b)

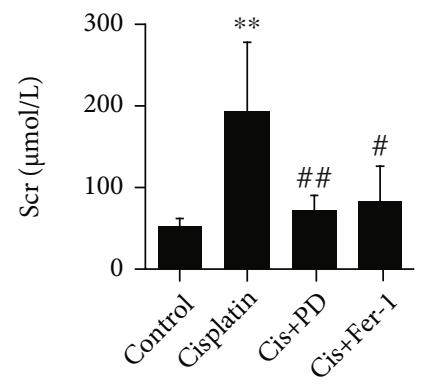

(d)

Cis+Fer-1
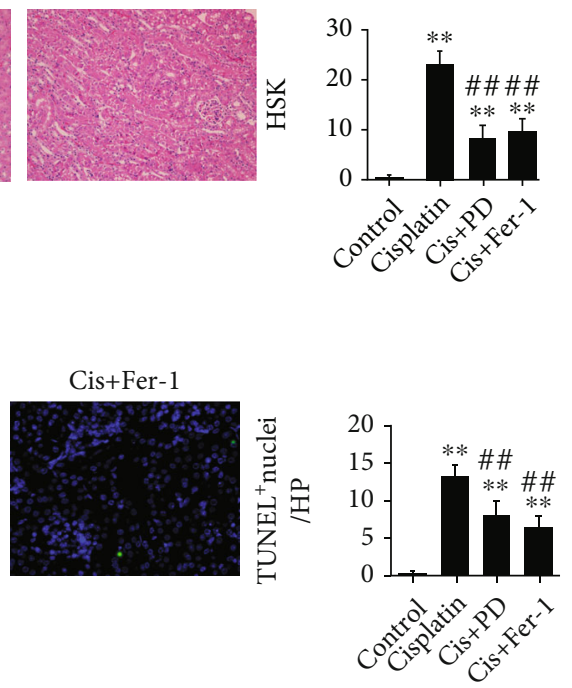

(f)

FIGURE 1: Therapeutical effects of PD on attenuating renal injury in Cis-AKI mice. (a) Mice pretreated with PD (40 mg/kg) or Fer-1 (5 mg/ $\mathrm{kg}$ ) were administered with intraperitoneal injections of cisplatin $(20 \mathrm{mg} / \mathrm{kg})$, and either PD or Fer-1 was intraperitoneally reinjected at $24 \mathrm{~h}$ after cisplatin injection. They were executed $48 \mathrm{~h}$ after the cisplatin injection. (b-d) Body weight changes, BUN, and Scr were measured at $48 \mathrm{~h}$ after injection of cisplatin. (e, f) Histopathology analysis of the kidneys in Cis-AKI mice was performed by hematoxylin-eosin (H\&E) staining (400x magnification), and the HSK was graded. (g) Representative terminal deoxynucleotidyl transferase dUTP nick-end labeling(TUNEL-) stained sections of the kidney (200x magnification). $* P<0.05, * * P<0.01$ vs. control; ${ }^{\#} P<0.05,{ }^{\# \#} P<0.01$ vs. cisplatin; ${ }^{\$} P<0.05,{ }^{\$} P<0.01$ vs. Cis + PD ( $n=5$ to 7 ). Cis: cisplatin; PD: polydatin; Fer-1: ferrostatin-1; BUN: blood urea nitrogen; Scr: serum creatinine; HSK: histological score of kidney injuries.

with nondrug-treated HK-2 cells. The effect of the $40 \mu \mathrm{M}$ dose of PD was more obvious than that of Fer-1 $(1 \mu \mathrm{M})$ and deferoxamine (DFO) $(100 \mu \mathrm{M})$ (Figure 3(a)).

The toxicity of cisplatin on HK-2 cells was detected by CCK- 8 assay, and the protective effect of PD was compared with that of Fer-1 $(1 \mu \mathrm{M})$ and DFO $(100 \mu \mathrm{M})$. Cisplatin
$(20 \mu \mathrm{M})$ exposure for $24 \mathrm{~h}$ significantly inhibited the viability of HK-2 cells compared with the control group (Figure 3(b)). Therefore, a dose of $20 \mu \mathrm{M}$ cisplatin was used in the subsequent experiments. The protective effect of $\mathrm{PD}$ on cell viability after cisplatin treatment was dose-dependent, with $40 \mu \mathrm{M}$ as the optimal concentration 

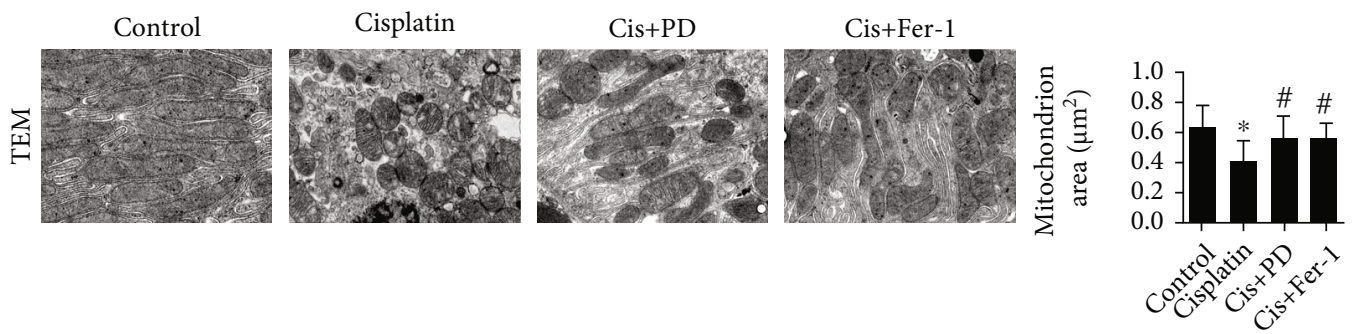

(a)
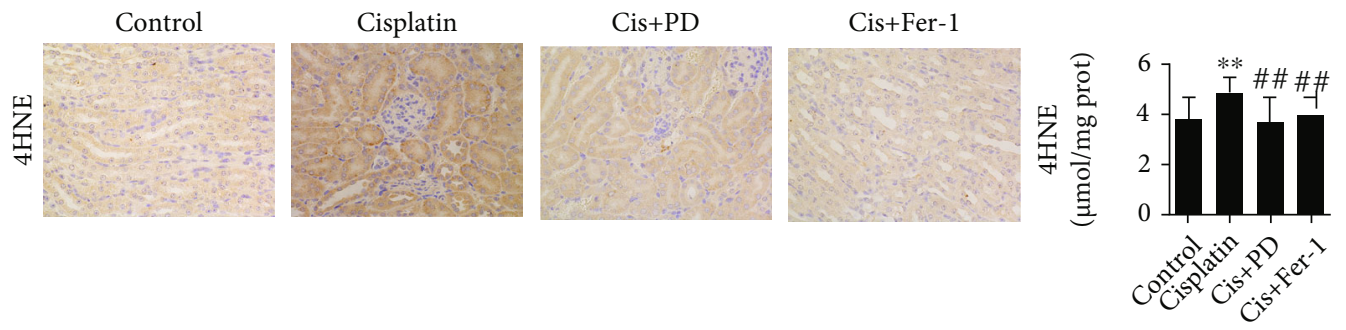

(b)

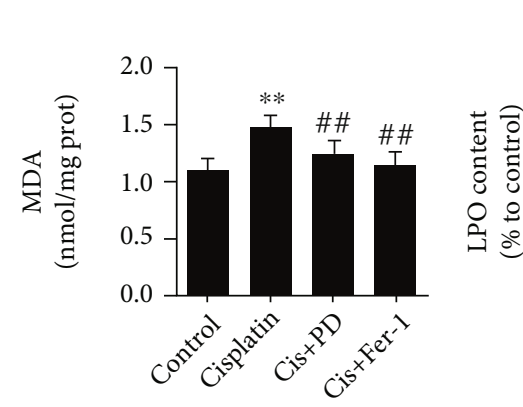

(c)

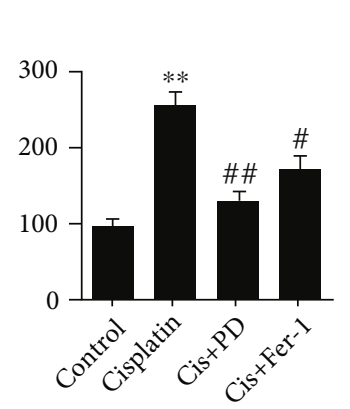

(d)

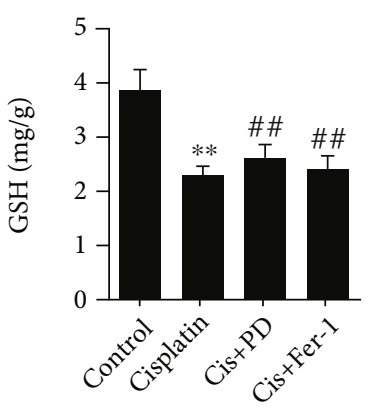

(e)

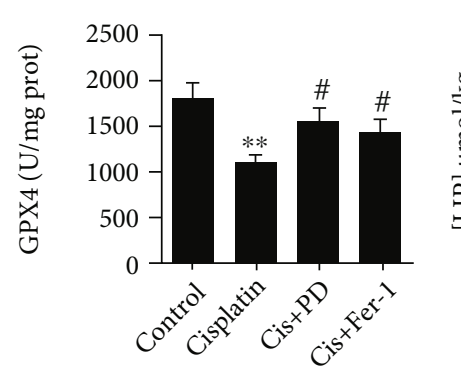

(f)

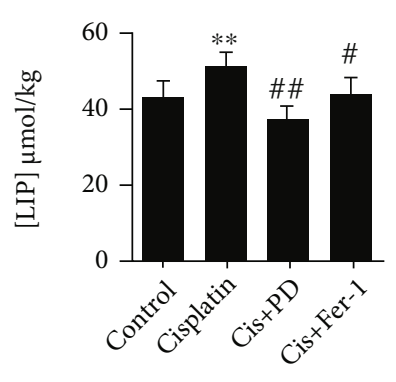

(g)

FIGURE 2: Effects of PD on inhibiting ferroptosis in Cis-AKI mice. (a) The ultrastructure of the renal tissue was seized by TEM at $48 \mathrm{~h}$ after cisplatin injection (12000x magnification). (b-d) The lipid peroxidation markers, immunohistochemistry for 4HNE (400x magnification), and determination of MDA and LPO were performed at $48 \mathrm{~h}$ after cisplatin injection. (e, f) The level of GSH and the activity of GPx4 were tested in kidneys at $48 \mathrm{~h}$ after cisplatin injection. $(\mathrm{g})$ Levels of chelatable iron in kidneys were determined by EPR detection of ferrioxamine. $* P<0.05, * * P<0.01$ vs. control; ${ }^{\#} P<0.05$, ${ }^{\# \#} P<0.01$ vs. cisplatin; ${ }^{\$} P<0.05,{ }^{\$ \$} P<0.01$ vs. Cis $+\mathrm{PD}$ ( $n=5$ to 7 ). Cis: cisplatin; PD: polydatin; Fer-1: ferrostatin-1; TEM: transmission electron microscopy; 4HNE: 4-hydroxynonenal; MDA: malondialdehyde; LPO: lipid hydroperoxide; GSH: glutathione; GPx4: glutathione peroxidase-4; LIP: labile iron pool.

(Figure 3(c)). Based on this result, $40 \mu \mathrm{M}$ PD was used for the subsequent experiments.

We also analyzed mitochondrial membrane potential (MMP) by florescent JC-1 in cisplatin-induced damage of HK-2 cells treated with or without PD or Fer-1, and the results showed that both PD and Fer-1 could reverse the mitochondrial damage induced by cisplatin (Figures 3(d) and 3(e)).
The intracellular labile iron and ferrous iron $\left(\mathrm{Fe}^{2+}\right)$ levels were detected using the FeRhoNox ${ }^{\mathrm{TM}}-1$ fluorescent probe (Figures 3(f)), $\mathrm{Fe}^{2+}$ assay (Figures 3(g)), and calcein AMchelatable LIP assay (Figure $3(\mathrm{~h})$ ). The results indicated a marked increase in intracellular labile iron and $\mathrm{Fe}^{2+}$ levels in HK-2 cells after cisplatin treatment, which was attenuated in PD-treated or Fer-1-treated cells. 


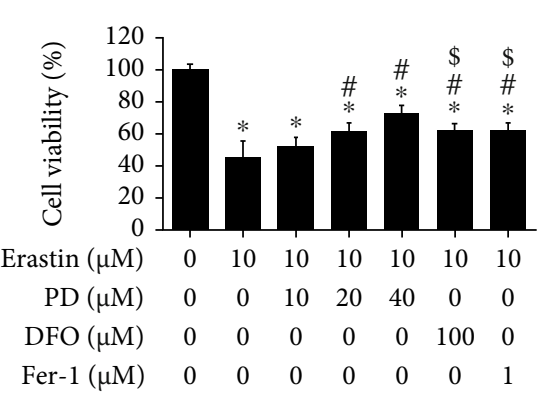

(a)

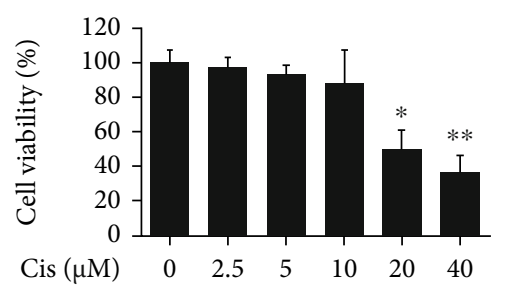

(b)

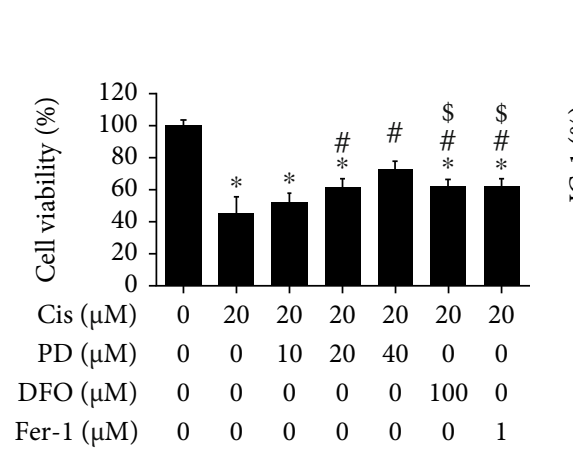

(c)

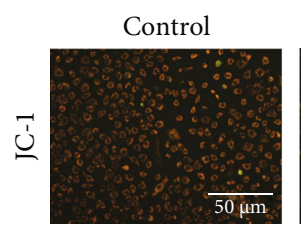

Cisplatin
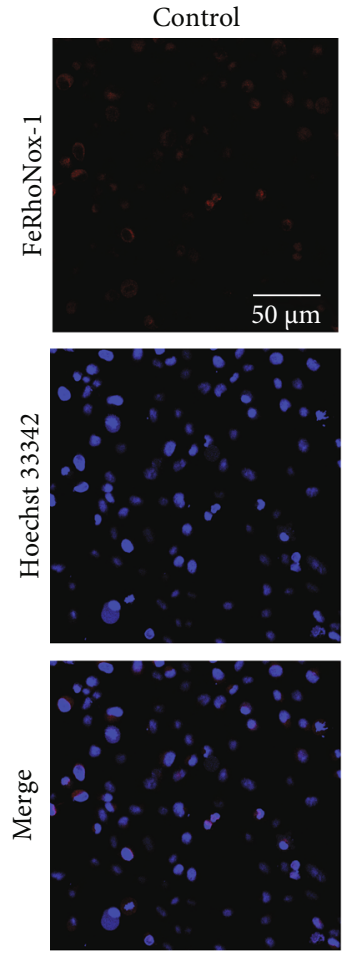

Cisplatin

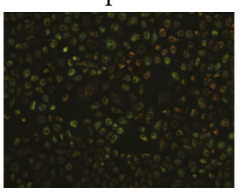

(e)
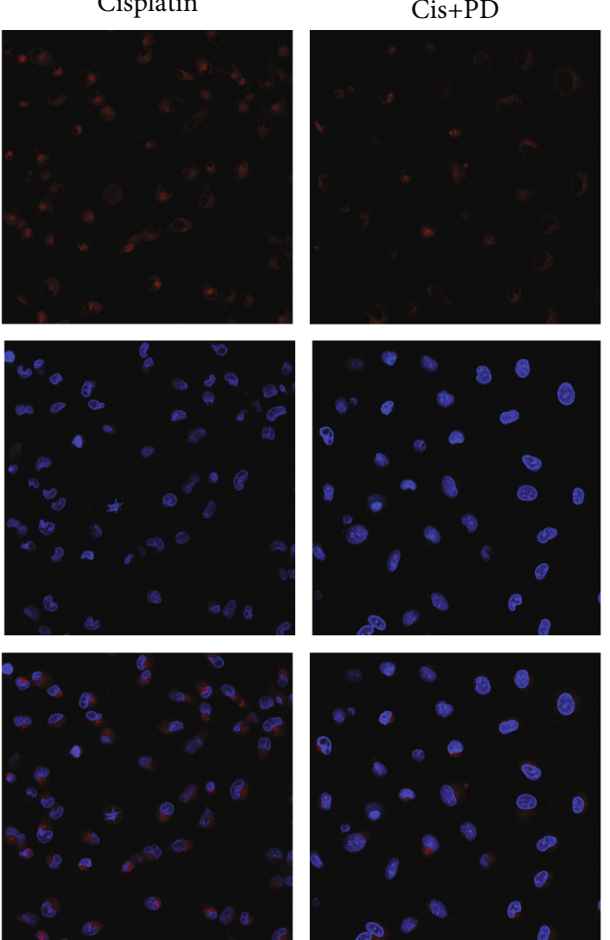

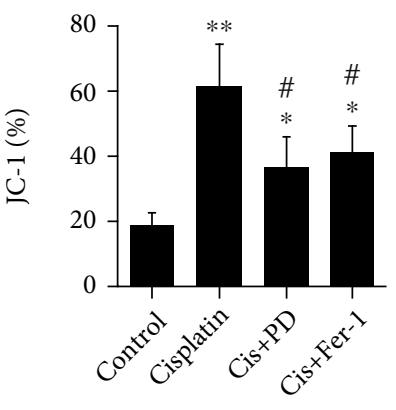

(d)

Cis $+\mathrm{PD}$

Cis + Fer-1
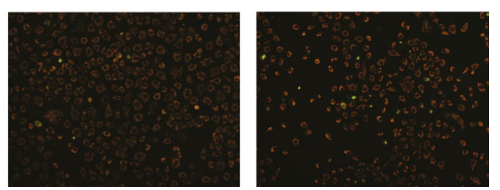

Cis+Fer-1
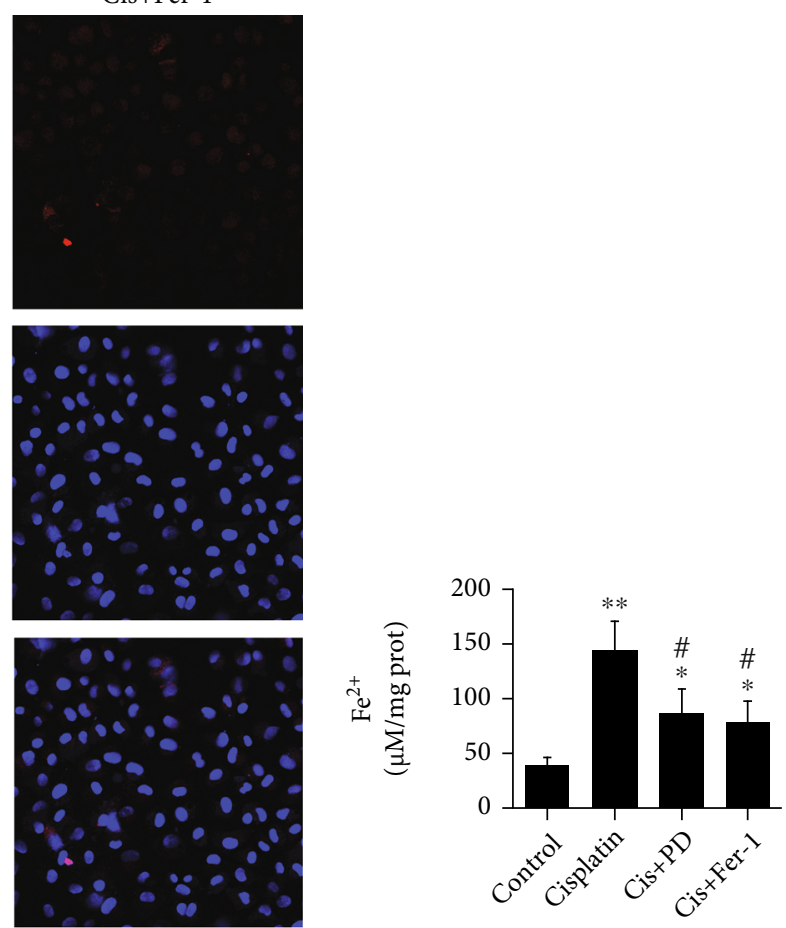

(g)

FIgURE 3: Continued. 


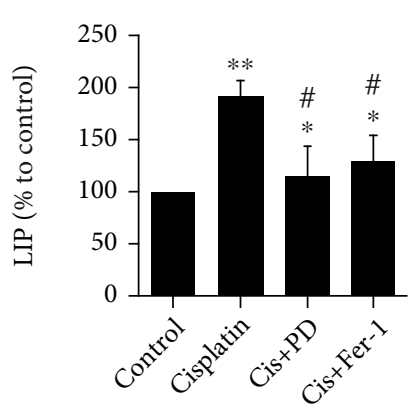

(h)

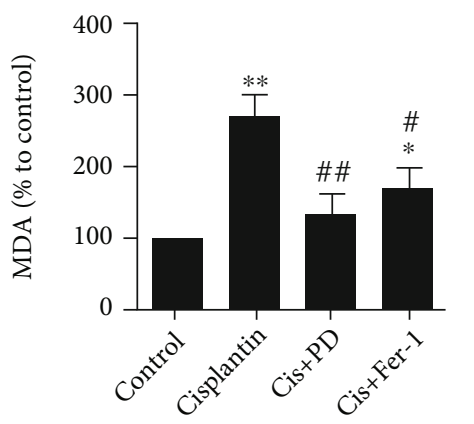

(j)

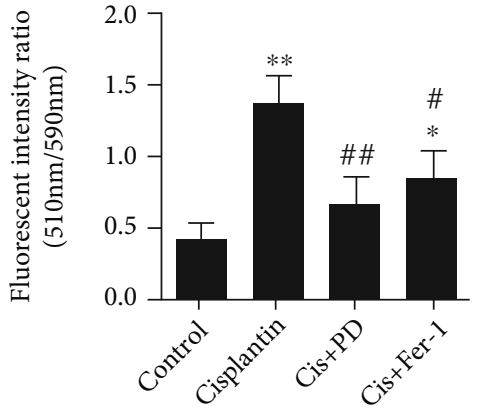

(i)

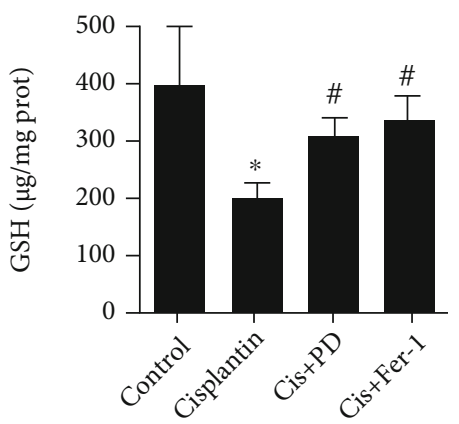

$(\mathrm{k})$

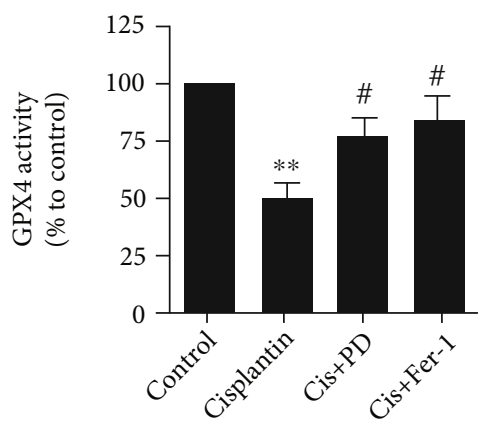

(1)

FIGURE 3: Inhibition of PD on cisplatin-induced ferroptosis in HK-2 cells. (a) CCK8 showing the viability of HK-2 cells under the treatment of different concentration of PD $(10,20$, and $40 \mu \mathrm{M})$, DFO $(100 \mu \mathrm{M})$, or Fer-1 $(1 \mu \mathrm{M})$ in presence or absence of erastin $(20 \mu \mathrm{M})$. (b) CCK8 showing the viability of HK-2 cells under the simultaneous treatment of different concentration of cisplatin for $24 \mathrm{~h}$. (c) CCK8 showing the viability of HK-2 cells under the treatment of different concentration of PD $(10,20$, and $40 \mu \mathrm{M})$, DFO $(100 \mu \mathrm{M})$, or Fer-1 (1 $\mu \mathrm{M})$ with or without cisplatin $(20 \mu \mathrm{M})$. (d, e) Changes in MMP expression levels were detected by fluorescence microscope and flow cytometry using JC-1. (f-h) Intracellular labile iron in HK-2 cells was measured using selective Fe (II) fluorescent probe FeRhoNox-1, iron assay kit, and LIP assay. (i,j) The lipid peroxidation in HK-2 cells was measured using C11-BODIPY581/591 and MDA assay. (k, l) The level of GSH and the GPx4 activity of HK-2 cells were examined in $24 \mathrm{~h}$ after cisplatin injection. $* P<0.05, * * P<0.01$ vs. control (nondrug treated); ${ }^{\#} P<0.05,{ }^{\# \#} P<0.01$ vs. cisplatin (or erastin) alone; ${ }^{\$} P<0.05,{ }^{\$ \$} P<0.01$ vs. Cis (or erastin) $+40 \mu \mathrm{M}$ PD ( $n=6$ ). Cis: cisplatin; PD: polydatin; Fer-1: ferrostatin-1; DFO: deferoxamine; LIP: labile iron pool; MDA: malondialdehyde; GSH: glutathione; GPx4: glutathione peroxidase-4.

Lipid peroxidation was confirmed by C11 BODIPY 581/ 591 staining (Figure 3(i)) and MDA assay (Figure 3(j)), and the results show that the lipid peroxidation in HK-2 cells was increased by cisplatin treatment, which could be rescued by $40 \mu \mathrm{M}$ dose of PD treatment, and the effect was even stronger than that of $1 \mu \mathrm{M}$ Fer- 1 .

The measurement of GSH level (Figure 3(k)) and GPx4 activity (Figure 3(1)) showed that both PD and Fer-1 treatment significantly mitigated the decrease in GSH level and GPx4 activity after cisplatin induction.
3.4. PD Limits Intracellular ROS Increase Induced by Cisplatin In Vitro and In Vivo. Excessive $\mathrm{Fe}^{2+}$ levels in cells induce a large amount of reactive oxygen free radicals, which further attack and oxidize cell membrane lipids by triggering ferroptosis. In this study, intracellular reactive oxygen species (ROS) were measured by using DCFH-DA fluorescent probes for cisplatin-induced HK-2 cells and DHE fluorescent probes for the kidneys of Cis-AKI mice. Compared with that of the control group, the ROS levels of HK-2 cells exposed to cisplatin significantly increased, 

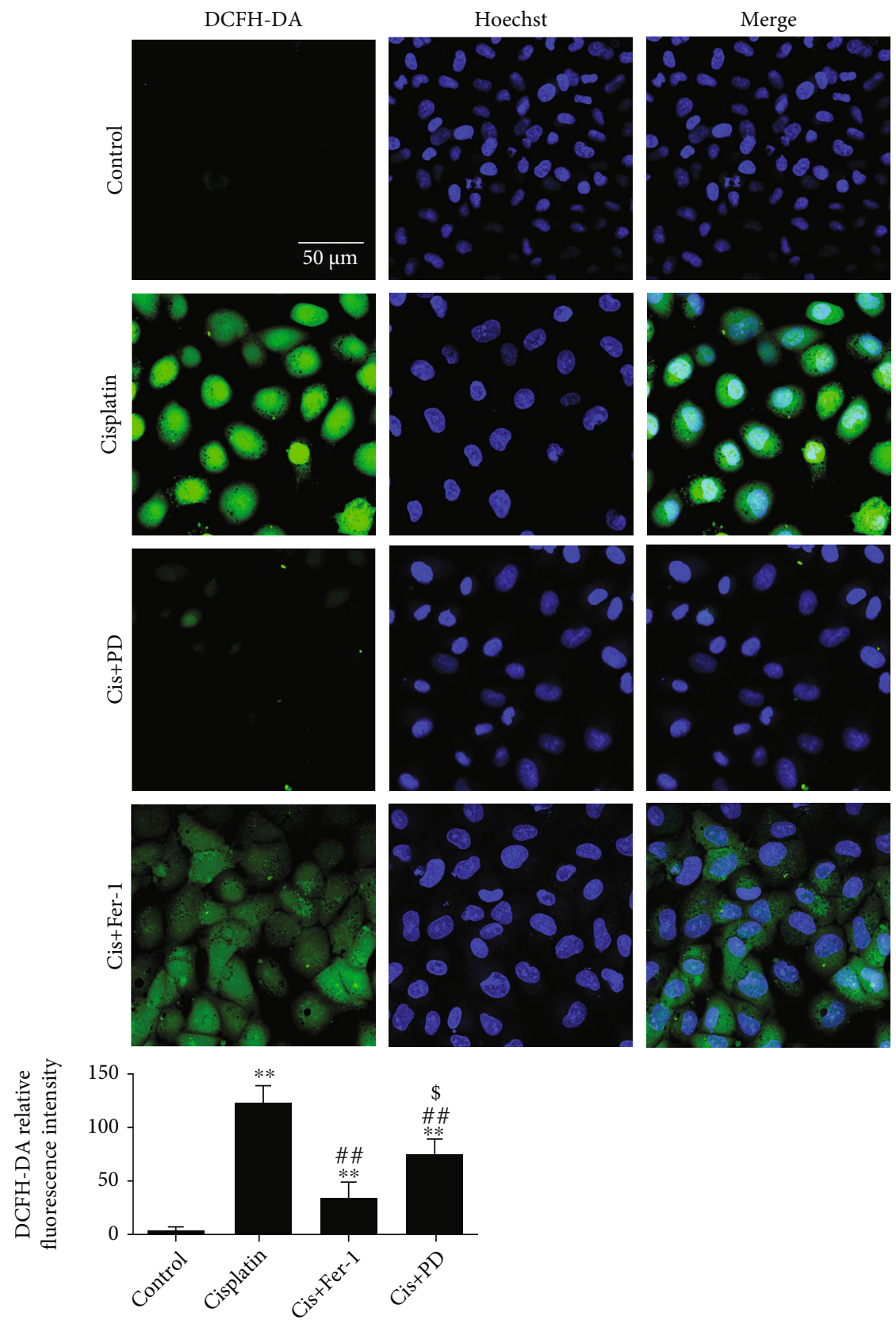

(a)

FIgUre 4: Continued. 

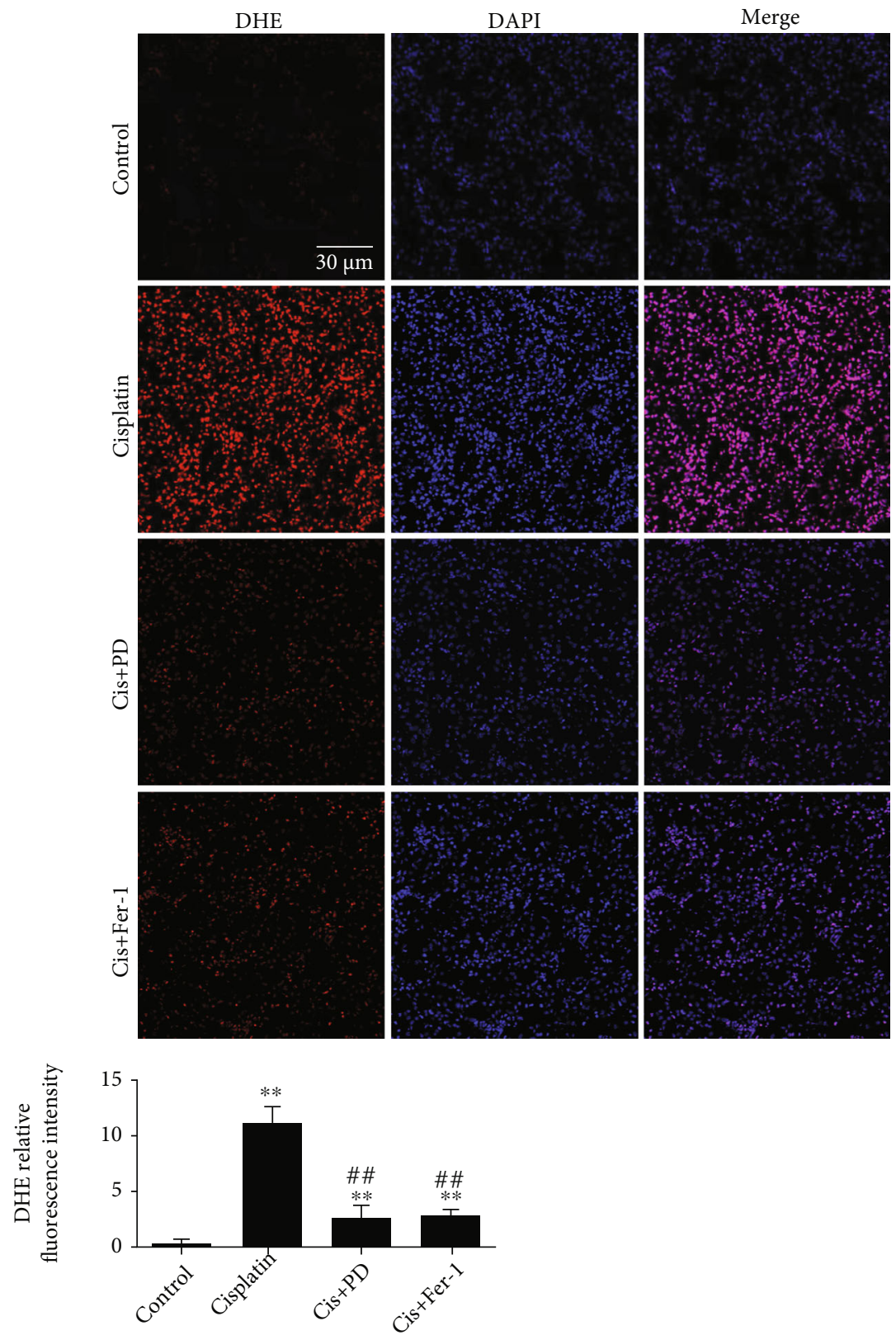

(b)

FIGURE 4: PD limits intracellular ROS increase induced by cisplatin in vitro and in vivo. (a) The ROS level of HK-2 cells was captured by CLSM at $24 \mathrm{~h}$ after cisplatin injection (DCFH-DA fluorescent staining, 600x magnification). (b) The ROS level of kidney tissues was captured by CLSM at $48 \mathrm{~h}$ after cisplatin injection (DHE fluorescent staining, 200x magnification). $* P<0.05, * * P<0.01$ vs. control; ${ }^{\#} P<0.05,{ }^{\# \#} P<0.01$ vs. cisplatin; ${ }^{\$} P<0.05,{ }^{\$ \$} P<0.01$ vs. Cis $+\mathrm{PD}(n=5-7)$. Cis: cisplatin; PD: polydatin; Fer-1: ferrostatin-1; DCFHDA: $2^{\prime}, 7^{\prime}$-dichlorofluorescin diacetate; DHE: dihydroethidium.

which decreased after supplementation with a $40 \mu \mathrm{M}$ dose of $\mathrm{PD}$, and the effect was even stronger than that of a $1 \mu \mathrm{M}$ dose of Fer-1 (Figure 4(a)).

Likewise, the level of intracellular ROS in kidneys measured by the fluorescent probe DHE was increased in Cis-AKI mice compared with that of the control and was ameliorated in PD $(40 \mathrm{mg} / \mathrm{kg})$-pretreated Cis-AKI mice, and its effect was almost comparable to that of Fer-1 $(5 \mathrm{mg} / \mathrm{kg})$ (Figure 4(b)).

\section{Discussion}

Recommended as a first-line chemotherapeutic, cisplatin is applied in the treatment of various types of malignancies, including lymphoma, germ cell carcinomas, and neoplasms of the prostate, bladder, and lung [1]. Cisplatin freely passes through glomerular filter and is largely reabsorbed by proximal tubules (especially S3 segment tubules), and such localized accumulation may result in nephrotoxicity [43]. As 
early as 30 years ago, researchers proposed that cisplatininduced free iron overload leads to lipid peroxidation in kidneys, but the link between ferroptosis and Cis-AKI has only recently been recognized. Denget al. [5] confirmed that cisplatin induced free iron release, GSH depletion, a decrease in GPx4 activity and excessive lipid peroxidation in vivo and in vitro, which was subsequently verified by other groups of investigators [2-4]. Ferroptosis, officially named in 2012, is characterized by intracellular iron accumulation and lipid peroxidation apart from classical apoptosis, necrosis, and autophagy [44]. Activating or inhibiting ferroptosis has become a new strategy for treating various diseases [45]. Exploring more potential nephroprotective compounds for ferroptosis will provide an experimental basis for their clinical treatment. In this study, we investigated the nephroprotective effect of PD and elucidated its possible antiferroptotic mechanism. Our innovative findings include defining abnormal activation of ferroptosis in Cis-AKI and demonstrating that $\mathrm{PD}$ contributes to protection against Cis-AKI by inhibiting ferroptosis through reducing excessive free iron accumulation and increasing GPx4 activity.

Studies using the ferroptosis inducer erastin have shown that the system $\mathrm{Xc}^{-}-\mathrm{GSH}-\mathrm{GPx} 4$ axis plays a central role in limiting lipid peroxidation and ferroptosis [46]. Lipid peroxidation due to GSH depletion is a key feature of ferroptosis. GPx4 is a lipid enzyme that can catalyze the conversion of GSH to glutathione disulfide (GSSG) in the oxidation reaction and then remove excess peroxides, subsequently alleviating the peroxidation of polyunsaturated fatty acids in the membrane [47]. Cisplatin has a high affinity for mercaptan-rich biomolecules, including GSH. In the cytoplasm, the majority of intracellular cisplatin is conjugated to GSH to form the Pt-GS complex [48]. Similar to erastin, depletion of GSH along with the inactivation of GPxs was the underlying mechanism of action for cisplatin. In recent years, several studies [49-55] found the nephroprotective effects of PD and its aglycone and resveratrol, against cisplatin-induced oxidative stress and inflammation. However, their effects on inhibiting ferroptosis have only recently been reported in myocardial ischemia-reperfusion injury and intracerebral hemorrhage models [36-38]. Our results identified that a $40 \mathrm{mg} / \mathrm{kg}$ dose of PD significantly rescued the depletion of GSH and the decrease in GPx4 activity after cisplatin induction, and its effect was almost equivalent to that of a $5 \mathrm{mg} / \mathrm{kg}$ dose of Fer-1. Erastin, a potent and selective inhibitor of system $\mathrm{Xc}^{-}$[56], can lead to GSH depletion and loss of GPx4 activity by preventing cystine import and then reduce the clearance of lipid peroxide and induce ferroptosis. In this study, we determined that PD dosedependently alleviated erastin-induced cell death in HK-2 cells, and $40 \mu \mathrm{M}$ PD has a significant inhibitory effect on ferroptosis. These results suggest that PD may exert a regulatory effect on the system $\mathrm{Xc}^{-}-\mathrm{GSH}-\mathrm{GPx} 4$ axis, which may be an important antiferroptotic mechanism of PD.

Ferroptosis is dependent on excessive accumulation of free iron $\left(\mathrm{Fe}^{2+}\right)$, which is a crucial component of lipid oxidation [45]. $\mathrm{Fe}^{2+}$ is partially transferred by ferritin and can be partly stored in the LIP. Abnormal iron homeostasis and excess intracellular $\mathrm{Fe}^{2+}$ levels lead to the production of a quantity of reactive oxygen free radicals, which further attack and oxidize cell membrane lipids, resulting in ferroptosis [45]. Resveratrol, an aglycone of PD, has been widely demonstrated to ameliorate organ damage caused by iron overload. A recent study noted that PD can dramatically reduce the deposition of iron in traumatic brain injury [36], although its mechanism was not discussed in detail. Iron chelators, including DFO, prevent free radical production and delay ferroptosis. In previous studies, polyphenols were often found to be potent iron chelators, and several polyphenol compounds have been reported to chelate iron and regulate iron homeostasis, such as proanthocyanidin, curcumin, quercetin, and silymarin [57-60]. In the present study, we verified that PD could significantly inhibit excessive intracellular $\mathrm{Fe}^{2+}$ accumulation and ROS generation both in the kidneys of Cis-AKI mice in vivo and in HK-2 cells after cisplatin induction in vitro. $\mathrm{PD}$, as a natural polyphenolic compound, has strong biological activity and remains to have unique potential to regulate iron metabolism, although its specific mechanism remains to be further clarified. This study confirmed that PD inhibits ferroptosis in cisplatin-induced AKI in vitro and in vivo, although PD requires higher dose than the traditional ferroptosis inhibitor Fer-1. PD has comprehensive effects, such as antioxidative, anti-inflammation, and autophagy-regulating effects, in addition to its inactivation of ferroptosis, as well as its superior potential for clinical application. However, we still propose that further exploration of the effect and mechanism of PD in AKI and chronic kidney disease will provide a new therapeutic option for all nephrology practitioners. Furthermore, it is not clear whether the action of PD might be referable to iron chelation, free radical scavenging, or other actions on iron handling.

\section{Conclusions}

In conclusion, in vitro and in vivo experiments indicated the prominent nephroprotective effects of PD against ferroptosis in Cis-AKI models, occurred at least partly through inhibiting excessive intracellular free iron accumulation and ROS production, rescuing GSH consumption, and enhancing GPx4 activity, thereby decreasing lipid peroxidation and ferroptosis sensitivity and ultimately attenuating the pathological progression of AKI. Although the specific molecular mechanism of PD against ferroptosis has still not been comprehensively determined, the current work might provide novel insight into the potential clinical application of PD as a drug for AKI treatment.

\section{Abbreviations}

AKI: $\quad$ Acute kidney injury

Cis-AKI: Cisplatin-induced AKI

GPx4: Glutathione peroxidase-4

PD: $\quad$ Polydatin

GSH: Glutathione

MDA: Malondialdehyde

DHE: Dihydroethidium

Fer-1: Ferrostatin-1 
DFO: Deferoxamine

ROS: $\quad$ Reactive oxygen species

4HNE: 4-Hydroxynonenal

LPO: $\quad$ Lipid hydroperoxide

BUN: Blood urea nitrogen

Scr: $\quad$ Serum creatinine

TUNEL: Terminal deoxynucleotidyl transferase dUTP nickend labeling

HSK: $\quad$ Histological score of kidney injuries

TEM: Transmission electron microscopy

EPR: Electron paramagnetic resonance

LIP: Labile iron pool

CLSM: Confocal laser-scanning microscope

MMP: Mitochondrial membrane potential.

\section{Data Availability}

All data related to this paper may also be requested from the corresponding authors (email: xjsnlhb@fmmu.edu.cn).

\section{Conflicts of Interest}

The authors declare that there is no conflict of interest regarding the publication of this paper.

\section{Authors' Contributions}

Lu Zhou, Peng Yu, Ting-ting Wang, Yi-wei Du, and Yang Chen contributed equally to this work.

\section{Acknowledgments}

This work was supported by grants from the Natural Science Basic Research Plan in Shaanxi Province of China (grant number 2019JM-033), Subject Platform and Technology Innovation Development Foundation of Tangdu Hospital (grant numbers 2019QYTS003, 2020XKPT014, and 2021QYJC-001), and National Natural Science Foundation of China (nos. 82070261 and 81803053).

\section{References}

[1] C. Y. Fang, D. Y. Lou, L. Q. Zhou et al., "Natural products: potential treatments for cisplatin-induced nephrotoxicity," Acta Pharmacologica Sinica, vol. 42, no. 12, pp. 1951-1969, 2021.

[2] Z. Hu, H. Zhang, B. Yi et al., "VDR activation attenuate cisplatin induced AKI by inhibiting ferroptosis," Cell Death \& Disease, vol. 11, no. 1, p. 73, 2020.

[3] D. U. Kim, D. G. Kim, J. W. Choi et al., "Loganin attenuates the severity of acute kidney injury induced by cisplatin through the inhibition of ERK activation in mice," International Journal of Molecular Sciences, vol. 22, no. 3, p. 1421, 2021.

[4] E. Mishima, E. Sato, J. Ito et al., "Drugs repurposed as antiferroptosis agents suppress organ damage, including AKI, by functioning as lipid peroxyl radical scavengers," Journal of the American Society of Nephrology: JASN, vol. 31, no. 2, pp. 280-296, 2020.

[5] F. Deng, I. Sharma, Y. Dai, M. Yang, and Y. S. Kanwar, "Myoinositol oxygenase expression profile modulates pathogenic ferroptosis in the renal proximal tubule," The Journal of Clinical Investigation, vol. 129, no. 11, pp. 5033-5049, 2019.

[6] Y. Ikeda, H. Hamano, Y. Horinouchi et al., "Role of ferroptosis in cisplatin-induced acute nephrotoxicity in mice," Journal of Trace Elements in Medicine and Biology, vol. 67, p. 126798, 2021.

[7] Z. Deng, M. Sun, J. Wu et al., "SIRT1 attenuates sepsis-induced acute kidney injury via Beclin1 deacetylation-mediated autophagy activation," Cell Death \& Disease, vol. 12, no. 2, p. 217, 2021.

[8] Y. Gao, X. Dai, Y. Li et al., "Role of Parkin-mediated mitophagy in the protective effect of polydatin in sepsis-induced acute kidney injury," Journal of Translational Medicine, vol. 18, no. 1, p. 114, 2020.

[9] Z. Sun and X. Wang, "Protective effects of polydatin on multiple organ ischemia-reperfusion injury," Bioorganic Chemistry, vol. 94, p. 103485, 2020.

[10] L. Gu, J. Liu, D. Xu, and Y. Lu, "Polydatin prevents LPSinduced acute kidney injury through inhibiting inflammatory and oxidative responses," Microbial Pathogenesis, vol. 137, p. 103688, 2019.

[11] S. Xu, Z. Zeng, M. Zhao et al., "Evidence for SIRT1 mediated HMGB1 release from kidney cells in the early stages of hemorrhagic shock," Frontiers in Physiology, vol. 10, p. 854, 2019.

[12] Z. Zeng, Z. Chen, S. Xu et al., "Polydatin protecting kidneys against hemorrhagic shock-induced mitochondrial dysfunction via SIRT1 activation and p53 deacetylation," Oxidative Medicine and Cellular Longevity, vol. 2016, Article ID 1737185, 15 pages, 2016.

[13] Q. H. Meng, H. B. Liu, and J. B. Wang, "Polydatin ameliorates renal ischemia/reperfusion injury by decreasing apoptosis and oxidative stress through activating sonic hedgehog signaling pathway," Food and Chemical Toxicology: An International Journal Published for the British Industrial Biological Research Association, vol. 96, pp. 215-225, 2016.

[14] H. B. Liu, Q. H. Meng, C. Huang, J. B. Wang, and X. W. Liu, "Nephroprotective effects of polydatin against ischemia/reperfusion injury: a role for the PI3K/Akt signal pathway," Oxidative Medicine and Cellular Longevity, vol. 2015, Article ID 362158, 13 pages, 2015.

[15] Q. Meng, H. Liu, and J. Wang, "Protective effects of polydatin on HK-2 cells against oxygen-glucose deprivation/re-oxygenation-induced injury by regulating Sonic hedgehog through PI3K/Akt signaling pathway," Xi bao yu fen zi mian yi xue za zhi $=$ Chinese Journal of Cellular and Molecular Immunology, vol. 31, no. 11, pp. 1452-1457, 2015.

[16] Y. Gao, Z. Zeng, T. Li et al., "Polydatin inhibits mitochondrial dysfunction in the renal tubular epithelial cells of a rat model of sepsis-induced acute kidney injury," Anesthesia and Analgesia, vol. 121, no. 5, pp. 1251-1260, 2015.

[17] A. M. Abd El-Hameed, "Polydatin-loaded chitosan nanoparticles ameliorates early diabetic nephropathy by attenuating oxidative stress and inflammatory responses in streptozotocin-induced diabetic rat," Journal of Diabetes and Metabolic Disorders, vol. 19, no. 2, pp. 1599-1607, 2020.

[18] T. T. Gu, D. M. Zhang, Z. Y. Wan et al., "Polydatin enhances glomerular podocyte autophagy homeostasis by improving Nrf2-dependent antioxidant capacity in fructose-fed rats," Molecular and Cellular Endocrinology, vol. 520, p. 111079, 2021. 
[19] Z. Q. Chen, X. H. Sun, X. J. Li et al., "Polydatin attenuates renal fibrosis in diabetic mice through regulating the Cx32-Nox4 signaling pathway," Acta Pharmacologica Sinica, vol. 41, no. 12, pp. 1587-1596, 2020.

[20] Z. Ni, L. Tao, X. Xiaohui et al., "Polydatin impairs mitochondria fitness and ameliorates podocyte injury by suppressing Drp1 expression," Journal of Cellular Physiology, vol. 232, no. 10, pp. 2776-2787, 2017.

[21] W. Gong, J. Li, Z. Chen et al., "Polydatin promotes Nrf2-ARE anti-oxidative pathway through activating CKIP-1 to resist HG-induced up-regulation of FN and ICAM-1 in GMCs and diabetic mice kidneys," Free Radical Biology \& Medicine, vol. 106, pp. 393-405, 2017.

[22] C. Chen, K. Huang, J. Hao et al., "Polydatin attenuates AGEsinduced upregulation of fibronectin and ICAM-1 in rat glomerular mesangial cells and _db/db_ diabetic mice kidneys by inhibiting the activation of the SphK1-S1P signaling pathway," Molecular and Cellular Endocrinology, vol. 427, pp. 45$56,2016$.

[23] K. Huang, C. Chen, J. Hao et al., "Polydatin promotes Nrf2ARE anti-oxidative pathway through activating Sirtl to resist AGEs-induced upregulation of fibronetin and transforming growth factor- $\beta 1$ in rat glomerular messangial cells," Molecular and Cellular Endocrinology, vol. 399, pp. 178-189, 2015.

[24] X. Xie, J. Peng, K. Huang et al., "Polydatin ameliorates experimental diabetes-induced fibronectin through inhibiting the activation of NF- $\kappa \mathrm{B}$ signaling pathway in rat glomerular mesangial cells," Molecular and Cellular Endocrinology, vol. 362, no. 1-2, pp. 183-193, 2012.

[25] P. Liao, Y. He, F. Yang et al., "Polydatin effectively attenuates disease activity in lupus-prone mouse models by blocking ROS-mediated NET formation," Arthritis Research \& Therapy, vol. 20, no. 1, p. 254, 2018.

[26] B. Han, M. Gong, Z. Li, Y. Qiu, and Z. Zou, "NMR-based metabonomic study reveals intervention effects of polydatin on potassium oxonate-induced hyperuricemia in rats," Oxidative Medicine and Cellular Longevity, vol. 2020, Article ID 6943860, 10 pages, 2020.

[27] L. Chen and Z. Lan, "Polydatin attenuates potassium oxonateinduced hyperuricemia and kidney inflammation by inhibiting NF- $\kappa$ B/NLRP3 inflammasome activation via the AMPK/ SIRT1 pathway," Food \& Function, vol. 8, no. 5, pp. 17851792, 2017.

[28] L. Chen, Z. Lan, Q. Lin et al., "Polydatin ameliorates renal injury by attenuating oxidative stress-related inflammatory responses in fructose-induced urate nephropathic mice," Food and Chemical Toxicology, vol. 52, pp. 28-35, 2013.

[29] C. Cremon, V. Stanghellini, M. R. Barbaro et al., "Randomised clinical trial: the analgesic properties of dietary supplementation with palmitoylethanolamide and polydatin in irritable bowel syndrome," Alimentary Pharmacology \& Therapeutics, vol. 45, no. 7, pp. 909-922, 2017.

[30] A. F. Peritore, R. D’Amico, M. Cordaro et al., "PEA/polydatin: anti-inflammatory and antioxidant approach to counteract DNBS-induced colitis," Antioxidants, vol. 10, no. 3, p. 464, 2021.

[31] E. Gugliandolo, R. Fusco, F. Biundo et al., "Palmitoylethanolamide and polydatin combination reduces inflammation and oxidative stress in vascular injury," Pharmacological Research, vol. 123, pp. 83-92, 2017.

[32] S. K. Das, W. Wang, P. Zhabyeyev et al., "Iron-overload injury and cardiomyopathy in acquired and genetic models is attenu- ated by resveratrol therapy," Scientific Reports, vol. 5, no. 1, p. 18132, 2015.

[33] S. K. Das, P. Zhabyeyev, R. Basu et al., "Advanced ironoverload cardiomyopathy in a genetic murine model is rescued by resveratrol therapy," Bioscience Reports, vol. 38, no. 1, 2018.

[34] S. K. Das, J. DesAulniers, J. R. Dyck, Z. Kassiri, and G. Y. Oudit, "Resveratrol mediates therapeutic hepatic effects in acquired and genetic murine models of iron-overload," Liver International, vol. 36, no. 2, pp. 246-257, 2016.

[35] B. O. Murillo Ortiz, A. R. Fuentes Preciado, J. Ramírez Emiliano, S. Martínez Garza, E. Ramos Rodríguez, and L. A. de Alba Macías, "Recovery of bone and muscle mass in patients with chronic kidney disease and iron overload on hemodialysis and taking combined supplementation with curcumin and resveratrol," Clinical Interventions in Aging, vol. Volume 14, pp. 2055-2062, 2019.

[36] L. Huang, S. He, Q. Cai et al., "Polydatin alleviates traumatic brain injury: role of inhibiting ferroptosis," Biochemical and Biophysical Research Communications, vol. 556, pp. 149-155, 2021.

[37] Y. Mo, L. Duan, Y. Yang et al., "Nanoparticles improved resveratrol brain delivery and its therapeutic efficacy against intracerebral hemorrhage," Nanoscale, vol. 13, no. 6, pp. 3827-3840, 2021.

[38] T. Li, Y. Tan, S. Ouyang, J. He, and L. Liu, "Resveratrol protects against myocardial ischemia-reperfusion injury via attenuating ferroptosis," Gene, vol. 808, article 145968, 2022.

[39] J. S. Lou, L. P. Zhao, Z. H. Huang et al., "Ginkgetin derived from_Ginkgo biloba_ leaves enhances the therapeutic effect of cisplatin via ferroptosis-mediated disruption of the Nrf2/ HO-1 axis in EGFR wild-type non-small-cell lung cancer," Phytomedicine, vol. 80, p. 153370, 2021.

[40] A. N. Woodmansee and J. A. Imlay, "Quantitation of intracellular free iron by electron paramagnetic resonance spectroscopy," Methods in Enzymology, vol. 349, pp. 3-9, 2002.

[41] T. Mukaide, Y. Hattori, N. Misawa et al., "Histological detection of catalytic ferrous iron with the selective turn-on fluorescent probe RhoNox-1 in a Fenton reaction-based rat renal carcinogenesis model," Free Radical Research, vol. 48, no. 9, pp. 990-995, 2014.

[42] J. Lee, J. H. You, D. Shin, and J. L. Roh, "Inhibition of glutaredoxin 5 predisposes cisplatin-resistant head and neck cancer cells to ferroptosis," Theranostics, vol. 10, no. 17, pp. 77757786, 2020.

[43] F. Deng, X. Zheng, I. Sharma, Y. Dai, Y. Wang, and Y. S. Kanwar, "Regulated cell death in cisplatin-induced AKI: relevance ofmyo-inositol metabolism," American Journal of Physiology. Renal Physiology, vol. 320, no. 4, pp. F578-f595, 2021.

[44] S. J. Dixon, K. M. Lemberg, M. R. Lamprecht et al., "Ferroptosis: an iron-dependent form of nonapoptotic cell death," Cell, vol. 149, no. 5, pp. 1060-1072, 2012.

[45] S. Li and Y. Huang, "Ferroptosis: an iron-dependent cell death form linking metabolism, diseases, immune cell and targeted therapy," Clinical \& Translational Oncology, pp. 1-12, 2021.

[46] K. Hadian and B. R. Stockwell, "SnapShot: ferroptosis," Cell, vol. 181, no. 5, pp. 1188-1188.e1, 2020.

[47] J. Liu, R. Kang, and D. Tang, "Signaling pathways and defense mechanisms of ferroptosis," The FEBS Journal, 2021.

[48] Y. Min, C. Q. Mao, S. Chen, G. Ma, J. Wang, and Y. Liu, "Combating the drug resistance of cisplatin using a platinum 
prodrug based delivery system," Angewandte Chemie (International Ed. in English), vol. 51, no. 27, pp. 6742-6747, 2012.

[49] A. Ibrahim, F. A. al-Hizab, A. I. Abushouk, and M. M. AbdelDaim, "Nephroprotective effects of benzyl isothiocyanate and resveratrol against cisplatin-induced oxidative stress and inflammation," Frontiers in Pharmacology, vol. 9, p. 1268, 2018.

[50] M. A. Darwish, A. M. Abo-Youssef, M. M. Khalaf, A. A. Abo-Saif, I. G. Saleh, and T. M. Abdelghany, "Resveratrol influences platinum pharmacokinetics: a novel mechanism in protection against cisplatin-induced nephrotoxicity," Toxicology Letters, vol. 290, pp. 73-82, 2018.

[51] Q. Hao, X. Xiao, J. Zhen et al., "Resveratrol attenuates acute kidney injury by inhibiting death receptor-mediated apoptotic pathways in a cisplatin-induced rat model," Molecular Medicine Reports, vol. 14, no. 4, pp. 3683-3689, 2016.

[52] Y. Cigremis, M. Akgoz, H. Ozen et al., "Resveratrol ameliorates cisplatin-induced oxidative injury in New Zealand rabbits," Canadian Journal of Physiology and Pharmacology, vol. 93, no. 8, pp. 727-735, 2015.

[53] A. M. Osman, S. A. Telity, S. A. Telity et al., "Chemosensitizing and nephroprotective effect of resveratrol in cisplatin -treated animals," Cancer Cell International, vol. 15, no. 1, p. 6, 2015.

[54] M. A. Valentovic, J. G. Ball, J. Mike Brown et al., "Resveratrol attenuates cisplatin renal cortical cytotoxicity by modifying oxidative stress," Toxicology In Vitro, vol. 28, no. 2, pp. 248257, 2014.

[55] S. Ince, D. Arslan Acaroz, O. Neuwirth et al., "Protective effect of polydatin, a natural precursor of resveratrol, against cisplatin-induced toxicity in rats," Food and Chemical Toxicology, vol. 72, pp. 147-153, 2014.

[56] S. J. Dixon, D. N. Patel, M. Welsch et al., "Pharmacological inhibition of cystine-glutamate exchange induces endoplasmic reticulum stress and ferroptosis," eLife, vol. 3, article e02523, 2014.

[57] Q. Niu, L. Mu, S. Li, S. Xu, R. Ma, and S. Guo, "Proanthocyanidin protects human embryo hepatocytes from fluorideinduced oxidative stress by regulating iron metabolism," Biological Trace Element Research, vol. 169, no. 2, pp. 174-179, 2016.

[58] Y. Jiao, J. T. Wilkinson, X. di et al., "Curcumin, a cancer chemopreventive and chemotherapeutic agent, is a biologically active iron chelator," Blood, vol. 113, no. 2, pp. 462-469, 2009.

[59] Y. Tang, Y. Li, H. Yu et al., "Quercetin prevents ethanolinduced iron overload by regulating hepcidin through the BMP6/SMAD4 signaling pathway," The Journal of Nutritional Biochemistry, vol. 25, no. 6, pp. 675-682, 2014.

[60] B. A. Moayedi Esfahani, N. Reisi, and M. Mirmoghtadaei, "Evaluating the safety and efficacy of silymarin in $\beta$-thalassemia patients: a review," Hemoglobin, vol. 39, no. 2, pp. 7580, 2015. 